\title{
جودة التعليم العالي في حالة الأزمة وما بعدها
}

\author{
فتحي محمد عثمان الرملي 1 \\ كلية العلوم - جامعة بنغازي
}

\section{الملتخص:}

تتناول هذه الورقة بعض المفاهيم الهامة للجودة، ومايتعلق بجودة التعليم العالي. كما تلخص هذه الورقة المشاكل التي تطرأ علي التعليم العالي بسبب الأزمات و ما ينتج عن هذه المشاكل، و تطرح ثلاث نماذج دولية شهدت أزمات و كيف تعاملت معها. النموذج الأول هو كولومبيا، التي لم تحسن التعامل مع الأزمة و منذ ستينات القرن الماضي و مازالت تعاني حتى الأن. و النموذج الثاني هو اليابان و التي أهتمت بجودة التعليم بشكل عام في المقام الأول فأصبحت دولة في مصاف العالم الأول و منبع الجودة في كل المجالات و النموذج الثالث هو روندا. ثم تقدم الورقة توصيات للخروج من الأزمة بأقل الأثار السلبية المصاحبة لما و التي غالبا ما تتحول إلي صراعات و نزاعات تستمر إلي ما بعد الأزمة لسنوات طويلة.

الكلمات المفتاحية: جودة الخلدمة - جودة التعليم العالي- الأزمات و النزاعات. 


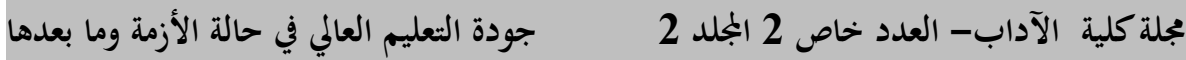

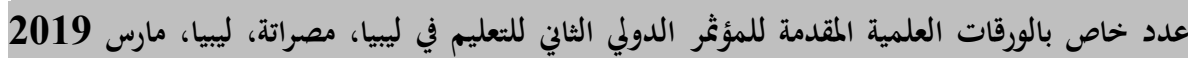

\section{Abestract}

This paper addresses some important concepts of quality, and is related to the quality of higher education. The paper also summarizes the problems that arise in education due to the crises and their consequences, and presents Three international models that have experienced crises and how they dealt with them. The first model is Colombia, which had not improved the handling of the crisis since the 1960s and it is still suffering. The second model is Japan, which is concerned with the quality of education in the first place, and had become a country in the top ranks of the world's and the source of quality in all fields. The third model is Rwanda. The paper then make recommendations out of the crisis with minimal adverse effects associated with them, which often turn into conflicts and disputes continue to post-crisis for years.

Keywords: Quality of service - quality of higher education - crises and conflicts.

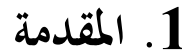

إن تحقيق التنمية المستدامة لا يمكن أن يحدث دون تعليم و بجودة عالية. لذلك فإن جودة التعليم العالي هي أمر ضروري و ليست من الترف و الكماليات. و قد نتفق بإن أفضل أستثمار هو الأستثمار في رأس المال البشري، و إن هدف إي أستثمار هو تحقيق ربح، السؤال الأن : ما هو الربح في حالة التعليم العالي ؟. إن الربح الحقيقي هو الحصول علي منتج وهو الخريج يحمل مهارات عالية و قدرات علمية ميزة. و بالطبع فإن هذا يعتمد علي العملية التعليمية التي يجب إن تتصف بالجودة لتكون مخرجاتما كذلك. 
في حالة جودة إنتاج يكون لدينا منتج نختبر جودته، فإذا كان المنتج صناعي مثلا فيكون له مقاييس و معايير مثل العمر الإفتراضي و الشكل الخارجي و المتانة ،الخ. و لكن في حالة التعليم العالي يكون الأمر ليس بسيط فقياس الجودة هنا له معايير اخرى.

\section{هنا تبرز بعض التساؤلات حول قياس الجودة :}

1. فهل حصول الطلبة علي تقديرات تلامس 100\% هو دليل علي ان التعليم العالي ذو جودة عالية؟

2. هل كثرة الذين يلتحقون بالجامعات يدل علي جودة التعليم العالي؟

و في حال الأزمات فإن التعليم العالي يتأثر شأنه في ذلك شأن كل القطاعات الأخرى، الاقتصادية والسياسية والأمنية والاجتماعية السائدة. بل قد يكون التعليم العالي أشدها حساسية للأزمات لإن مكوناته الأساسية هي الشباب. و في وجود الأزمات مثل الصراعات والحروب الأهلية و غيرها فإن جودة التعليم العالي تكون أول المتضررين، و ذلك لتضرر المباني والبنية التحتية للمؤسسات التعليمية و كذلك وتك ولك الهجرة و التهديدات الأمنية المختلفة. و تتكون حلقة قد لا تنتهي، ففشل التعليم العالي يؤدي إلي الألتحاق بالمحموعات المسلحة، و الألتحاق بالمحموعات المسلحة يقود إلي ترك فئك

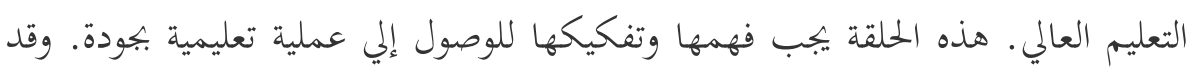
تستمر التأثيرات السلبية للأزمة إلي ما بعد زوالها. هذه الورقة تقدم تعاريف لجودة التعليم العالي و تضع أمام القارئ نموذجين لدولتين، إحداهما لم تلتفت إلي جودة التعليم العالي فأستمرت أزمتها لعقود طويلة ومازالت تعاني

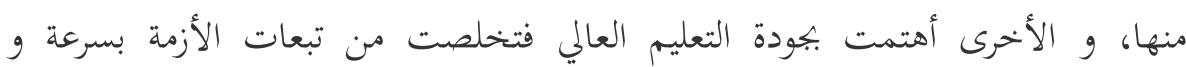
تحصلت علي مكاسب بشرية و مردودات مادية تفوق الدول التي لم تعاني إزمة. 
تعددت التعاريف التي أوردها المهتمين بموضوع الجودة في وضع تعريف محدد لمعنى ومضمون الجودة و أبعادها المختلفة. و هنا نذكر بعض التعريفات التي وضعت من بعض المختصين كتعريف للجودة. في قاموس أكسفورد الجودة تعني الدرجة العالية من النوعية والقيمة الجودة تعني تلبية متطلبات المستفيد و بجاوز توقعاته

يعرف جوزيف جوران (Juran, 1986) - اخصائي أمريكي في الإحصاء و الإدارة الجودة علي أها "الملائمة للإستخدام" - Fitness of Use. والمقصود بها بإن يكون مستخدم الخلدمة او السلعة قادرا علي الإعتماد عليها في تحقيق ما يريدها منها. الجودة هي الوفاء بالمتطلبات - إنعدام العيوب - تنفيذ العمل بصورة صحيحة من أول مرة و في كل مرة. "الجودة هي تحقيق احتياجات و توقعات المستفيد حاضرا ومستقبلال "Deming, $.1972)$ فيليب كروسبي (Crosby, 1979) و هو مؤسس كلية الجودة في أمريكا فيعرف الجودة بإها : "التطابق مع الإحتياجات و المواصفات" يعني بهذا إن جودة المنتج او اللندمة تنحصر في مدى مطابقته للمعايير الموضوعة.

و عموما فإن المفهوم القديم للجودة هو خلو الخدمة أو المنتج من العيوب، أما المفهوم الحديث ينطلق من مبدأ تلبية متطلبات المستفيد و بتحاوز توقعاته. 


\section{3. - 3 الجودة في التعليم العالي:}

مما لا شك فيه فإن الجودة تلعب دور مهم جدا في التعليم العالي و خصوصا في التعليم العالي، و يرى الكثيرون بأن الجودة في التعليم العالي تعتبر عامل رئيسي ومهم في التنافس و قياس التقدم بين الدول و إذها قوة اقتصادية مؤثرة جدا في اقتصاد كل الدول. و يعتقد ترابوس (Tribus, 1994) إنه"يجب إن نتذكر دائما الإختلاف بين الجودة في بحال التعليم العالي والجودة في مجال العمل و الإنتاج، و من هذه الاختلافات:

$$
\begin{aligned}
& \text { المؤسسة التعليمية ليست مصنع } \\
& \text { تعليم الطلاب ليس إنتاج } \\
& \text { الطالاب ليسوا منتجات }
\end{aligned}
$$

تحقيق الجودة يتطلب مشاركة الطلاب في العملية التعليمية، كعمال في بحال العمل.

لذلك فإن مفهوم الجودة في التعليم العالي لا يمكن صياغته بسهولة بتعريف محدد، فهو يختلف بإختلاف الروى والأهداف. و هنا نسوق بعض التعريفات التى نعتقد بإها تقدم تفسير مقبول لمعنى الجودة في التعليم العالي وآخرون يعرفوها بمعنى الإتقان الدائم في مخرجات التعليم العالي، و هناك من يعرفها علي إها القدرة علي التوفيق بين مخرجات التعليم العالي وسوق العمل، و من يستخدمها كمؤشر للمقارنة بين قيمة الناتج التعليمي و حجم الإنفاق علي التعليم العالي أو كمؤشر لقياس العائد المادي و غير المادي مقابل الإستثمار في التعليم العالي.

مفهوم جودة التعليم العالي يُقصد بها بحموعة المعايير والإجراءات التي يهدف تنفيذها إلى تحسين البيئة التعليميّة، بحيث تشمل هذه المعايير المؤسسات التعليميّة بمستوياتما 
وأشكالها المختلفة، والهيئة التدريسيّة والإداريّة وأحوال الموظفين الذين لهم علاقة مباشرة أو غير مباشرة بالمنظومة التعليميّة. معايير جودة التعليم العالي تختلف من مؤسسة إلي اخرى و لكنها تلتقي في المباديء الأساسية و التي تحدف جميعها إلي منتج بجودة عالية و هو الطالب.

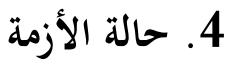

و نقصد بحالة الأزمة في ورقتنا هذه بإنها هي الحالة التي تنشأ بسبب ظروف قاهرة و هي حالة غير إعتيادية تتطلب التدخل لإدارتا و حلحلتها للعودة إلي الوضعية الطبيعية. و قد عرفها الكثيرون في بحالات متعددة. و نحن هنا يهمنا تعريفها في حالة التعليم العالي، و هي الإضطراب الذي يحدث في مسار التعليم العالي بسبب ظروف تكونت بسبب حالة طارئة تمر بها البلاد و تؤثر بشكل سلبي علي التعليم العالي. و قد تكون هذه الحالة بسبب الحروب او الكوارث الطبيعية او غيرها. و نعرف هنا الأزمة في التعليم العالي بأها حالة صعبة تحول دون تحقيق الأهداف الأساسية من العملية التعليمية.

\section{5. جودة التعليم العالي في ظل الأزمات}

يتأثر التعليم العالي شأنه في ذلك شأن كل مناحي الحياة الأخرى، الإقتصادية و السياسية و الأجتماعية، نتيجة الأزمات و النزاعات المحلية والإقليمية. و بالتالي يترتب علي

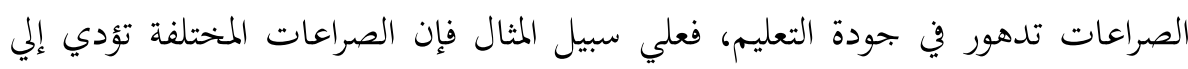
دمار المباني التعليمية و البنية التحتية، و هجرة العقول إلي الخارج و عموم الفوضى نتيجة إنتشار السلاح و غياب الأمن و نقص التمويل و غيرها من الأسباب التي سوف تؤثر

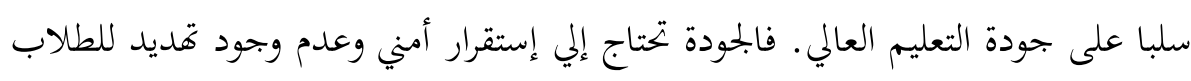


أو لإعضاء التدريس أو للعاملين و الإ فإن ظهور مشاكل و التى قد تصبح مزمنة هو نتيجة أكيدة و طبيعية.

\section{6. مشاكل و معوقات التعليم العالي بسبب الأزمات}

تظهر العديد من المشاكل و التي قد ترتقي لتصبح أمراض تصيب جسد التعليم

العالي و تتؤثر سلبيا على جودته و بالتالي على مخرجاته. و هذه الأمراض من الممكن أن توجد حتى في المجتمعات المستقرة و لكنها تصبح ظاهرة في حالات الإضطرابات و الأزمات و تستفحل مع أستمرار الأزمة حتى تدمر كل اركان جودة التعليم العالي. وهنا سوف نلخص بعض هده المشاكل . 1. التهديد الأمني: مثل إنتشار السلاح وتواجد جماعات مسلحة داخل الحرم التعليمي و عدم السيطرة علي الطلبة مما يؤدي إلي تحكم الطلبة استخدام التهديد للقائمين علي العملية التعليمية من أعضاء هيأة تدريس و عاملين وإدارة. و بالتالي تفشي الخوف. 2. ظاهرة الغش : لعدة أسباب قد تتفشى ظاهرة الغش و قد تتطور لتصبح غش جماعي، و بمعرفة حتى لجان الأشراف علي سير الإمتحانات و من الممكن أن ترتقى لتكون حتى بعلم المسؤولين علي التعليم العالي. و يكون ذلك بدوافع مثل الخوف من الطلاب و عدم السيطرة عليهم و إن بعض الطلاب ينتمي لجماعات مسلحة او مجموعات إجرامية. و يحاول البعض خلق تبريرات مثل يجب مراعاة ظروف الشباب والحالة التي شهدوها خلال الحرب. و لكن هذه المبررات غير صحية و لا تخدم جودة التعليم العالي. فمن يعاني من حالة نفسية، يجب علاجها و إعادة تأهيله بحيث لا يؤثر سلوكه علي البمتمع و لا علي التعليم العالي. 
3. الفساد الإداري والمالي: تشهد مراحل الصراع نمو لظواهر خطيرة مثل الرشوة و

المحسوبية و إباحة المال العام وغيرها. صحيح إن هذه المشاكل تكون موجودة حتى قبل الصراع و لكن سوف تزداد شراسة في حال الأزمات و تصبح أكثر ضرر مع وجود المشاكل الأخرى.

4. تسرب الامتحانات : و يكون ذلك احيانا بسبب الخوف من التهديدات او بسبب الحاجة الإقتصادية التي بسببها يقوم البعض ببيع الامتحانات. 5. تقليص المناهج : الوعاء الزمنى يكون قصير وذلك بسبب الإضطرابات و الصراعات التي تعوق وصول الطالب إلي مقر التعليم. و قد تتؤي إلي تأخر بدء العام الدراسي و كثرة التوقفات، و هذا يجبر القائمين علي التعليم العالي إلي تقليص محتويات المنهج مما يسبب نقص في المعلومات المهمة التي تعتمد عليها السنوات التالية. 6. عزوف الطلبة عن الحضور: و هذا بسبب حاجة بعضهم للعمل في ظل الظروف الإقتصادية الناشئة من الأزمة. أو التحاق بعضهم بمجموعات مسلحة. كذلك بسبب

$$
\text { نزوح البعض بحثا عن ملاذ أمن. }
$$

7. التأثيرات النفسية: يصاحب الأزمات المختلفة، سواء سياسية او إقتصادية أو غيرها، أزمات نفسية تكون مؤثرة بشكل كبير. و قد تتحول إلي حالات إنتقام من بعض

$$
\text { الطلبة و تنتشر علي أثرها حالات الظلم و اللامبالاة . }
$$

8. التسيب الإداري: لوجود إدارة ضعيفة او غير قادرة علي السيطرة يكثر تغيب الموظفين و الإداريين عن العمل و يصبح الإهمال هو الأمر السائد داخل اروقة الإدارات. و تتعطل الأعمال الضروية ويصبح طابع التأجيل و المماطلة هو أمر إعتيادي و مع الوقت يصبح أمر مقبول و طبيعي. 
9. التعليم العالي الخاص: إنتشار المؤسسات التعليمية الخاصة و التي لا تمدف أساسا إلي تعليم الناس و لكن هدفها هو الربح المادي، الأمر الذي تغيب معه الجودة و يكون الهدف هو العائد المادي السريع وليس مخرجات بجودة عالية.

\section{و قد ينتج عن المشاكل اعلاه ما يلي :}

1. فشل التقييم الحقيقي للطلاب وعدم تحديد الفروقات العلمية بينهم لحصول اغلبهم علي درجات عالية غير واقعية، الأمر الذي يؤدي إلي صعوبات تحقيق رغبات الطالب المتميز في دخول المحال الذي يناسبه. 2. حصول الطالب علي درجات عالية و لا تناسب مستواه العلمي، و نسبة النجاح قد تكون عالية و غير واقعية.

3. إهمال جماعي من أعضاء هيأة التدريس و الموظفين و الإدارة علي كل الأصعدة

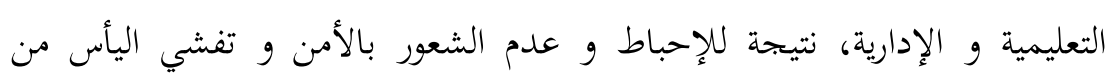
الإصلاح

4. بيئة غير مناسبة للتعليم من حيث المباني و الجو العام و غياب الأماكن المناسبة للتعليم مثل الأماكن الهادئة و المستقرة. 5. مخرجات عليا غير ذات كفاءة علمية و لا تتصف بأخلاق علمية.

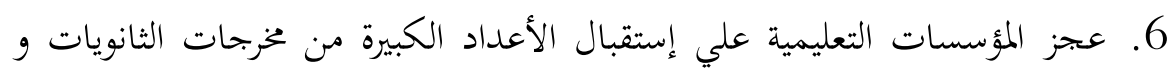
التي تحصلت علي تقادير عالية وهمية. 7. إستهلاك الوقت في تخصصات غير مناسبة لمستوى الطالب المنسب إليها و إهدار الأموال في غير محلها 8. كثرة التنقل بين الكليات لفشل بعض الطلاب في كليات و تخصصات أعلى من

$$
\text { مستوياتم الحقيقية. }
$$$$
\text { 9. إهيار في كل الجوانب علي مستوى الدولة. }
$$ 
الأن السؤال الذي يطرح نفسه ما الحل و ما العمل في حال حدوث الأزمة؟ و كيف

يمكننا إنفاذ ما يمكن إنقاذه من جودة التعليم العالي؟

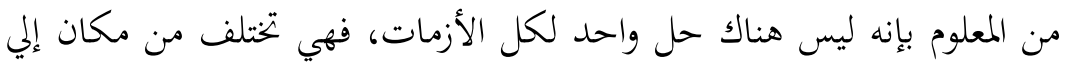

اخر ومن زمان إلي اخر. و لكن من المسلمات و البديهيات هو ضرورة سرعة تشكيل إدارة

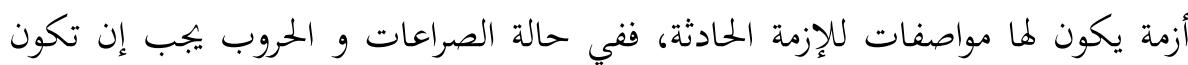
إدارة الأزمة قوية و بعيدة عن التجاذبات السياسية التى تطرأ نتيجة للصراع، و منها تشكل فرق عمل تعالج المشاكل التي تظهر بل و تكون لها القدرة علي توقع المشكلة حتى قبل ظهورها، و هذا يتطلب فرق عمل مدعومة بكل شيء و لها المرونة و القدرة علي الإستجابة السريعة و وضع الحلول المرحلية للخروج من المختنق او المشكل. لإن هذه الورقة

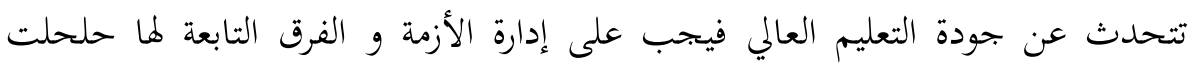
المشاكل التى ذكرناها في الأعلى و تفادي إنتشارها.

ما بعد الأزمة : كما ذكرنا سابقا من إن المشاكل التي تظهر مع الصراعات تستفحل و قد

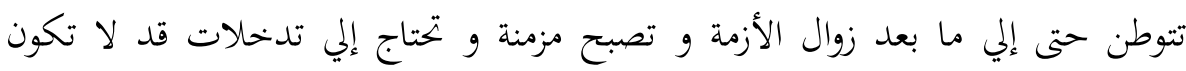
سهلة. و نرى إنه من المفيد هنا تقديم بتحارب لدول عانت من الحرب و والصراع المسلح لنستخلص منهما العبر و نستفيد من الإيجابيات و نتفادى السلبيات لنصل بالتعليم العالي إلي جودة مرجوة و نحقق مستوى جودة التعليم العالي.

النموذج الكولومبي

هذه الفقرة تقدم ملخص تاريخي لتحول طلبة جامعات في كولومبيا إلي مليشيا مسلحة و التي استمرت لعقود طويلة و مازالت مستمرة حتى الإن، هذا السرد لتوضيح بإن إهمال الجودة في التعليم يؤدي إلي ما لا يحمد عقباه. 
في الحالة الكولومبية يوجد العديد من الدراسات التى بحثت في العلاقة بين الحرب الأهلية التي شهدةًا كولومبيا و التعليم العالي. ما يهمنا هنا هو الحديث عن بحموعة مسلحة "تشكلت من طلبة الجحامعات الكولومبية في جزء من كولومبيا (Santander) في ستينات القرن الماضي. هذه المحموعة سمت نفسها جيش التحرير الوطني (ELN) و التي تكونت بتأثير من الثورة الكوبية في ذلك الوقت. بدأت المجموعة عملياتا في عام 1962 و ذلك بمجموعة مركزية تكونت من 16 شخص" (Soler, 2017). و قد قاد هذه المليشيا قس من الكنيسة الكاثولوكية في أمريكا اللاتينية. تلقت هذه المليشيا الدعم الشعبي بسبب رفعها لشعارات، مثل تخليص الشعب من حكم الأقلية المسيطرة إنذاك و محاربة الفقر و القضاء علي التبعية للإمبريالية الأمريكية. و في الثمانينات أصبحت هذه البمموعة تبحث عن مصارد تمويل فلجأت إلي سلب وإبتزاز الشركات الأجنيبة العاملة في كولومبيا. حاليا تعتبر ELN تعتبر ثاني أكبر عصابة مسلحة في كولومبيا و مازلت تستخدم السلب للتمويل كما تتحصل علي المال من قريب المخدرات و الخطف لطلب الفدية. و مازالت هذه المحموعة تسبب الدمار في البنية التحتية للبلاد و مستمرة في أعمال العنف ضد المدنيين و القوة الحكومية (Soler, 2017). إن الجحودة في التعليم العالي وتوفرها في حالة الأزمة و ما بعدها تقطع الطريق علي من يستفيد من حالة الإنفلات الأمني لتجنيد الطلاب و تحويلهم إلي بيادق وجنود و مع مرور الوقت يتحولون إلي عصابات إجرامية و أدوات لتنفيذ مخططات و أجندات. لذلك كان لزاما المحافظة علي جودة التعليم العالي و بصورة اكبر في حالة الأزمات و ما بعدها، و جعل المؤسسات التعليمية دور للبحث العلمي ومنابر للمعرفة و نشر العلم.

\section{النموذج الياباني}

بعدما أعتقد الكثيرون إن هضة اليابان بعد ما أهكتها الحرب شبة مستحيلة، و إن إستعادها بعض ما كان لديها من العلوم والثقافة هو ضرب من الخيال، و هي الدولة 
الوحيدة في العالم التي تلقت قنبلتين نوويتين و حرب شرسة، إنها الجزيرة التي لا تملك ثروات

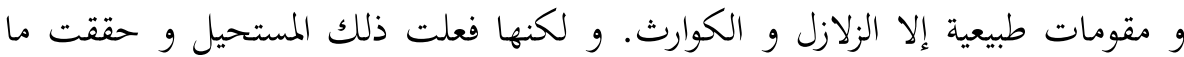
كان خيال. ماذا فعلت اليابان لتحقق ذلك؟ إن أول ما قامت به اليابان بعد الحرب هو الإهتمام بالجودة علي كل الإصعدة و جعلتها علي رأس الاولويات. فأصبحت اليابان قبلة لعلماء و مريدي الجودة و المهتمين بها. و كان من بينهم العالم ادوارد ديمنج، الذي يعد من أوائل الذين وضعوا الأسس العلمية والتطبيقية لإدارة الجودة الشاملة، و يعد مدخله الأكثر شيوعا واستخداما حيث ركز علي التحليل الإحصائي ومعايير قياس الجودة و قد وضع أربعة عشر نقطة كمبادئ للجودة الشاملة، أعتبرها البعض على أها نموذج لإدارة الجودة الشاملة، وقد عرف ديمنج باهتماماته الخاصة في بجال الإحصاء ودوره الهام عام ( 1950 ) كمعلم لليابانيين على كلى عمليات الرقابة الإحصائية .وقد أكد ديمنج في نقاطه الأربعة عشر علي الحاجة الماسة إلي طرق المراقبة الإحصائية والتعليم والانفتاح والهادف والتحسين المستمر والمشاركة، وفيما يلي مبادئ ديمنج في إدارة الجودة الشاملة(Deming, 1972) : 1. 1 وضع أهداف ثابتة لتحسين المخرجات 2. تبني فلسفة جديدة تتماشى مع التغييرات 3. بتحنب الاعتماد علي الفحص والتفتيش كوسيلة للحصول علي الجودة

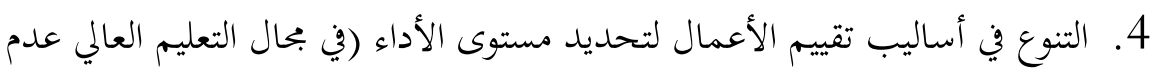
الاعتماد علي درجات المتعلم فقط).

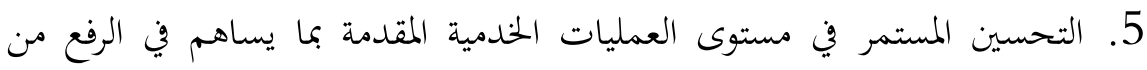
مستوى النوعية وتقليل التكلفة. 6. وضع برامج للتدريب أثناء الخدمة (التعليم مدى الحياة). 
7. إيجاد القيادة الفعالة.

8. البعد عن سياسة التخويف ليتمكن كل فرد العمل بكفاءة وإيجابية داخل المؤسسة. 9. كسر الحواجز بين الأقسام و تشكيل فرق عمل في مختلف الإدارات.

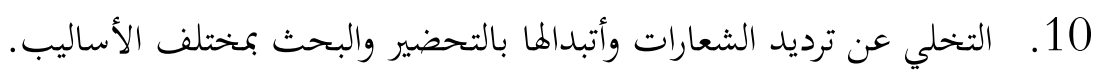
11. ت نقلق ثقافة الجودة. 12. 12 تحسين العلاقات داخل البيئة المؤسسية (إدارة ومعلمين ومتعلمين). 13. تشجيع الأفراد علي الفخر بما أبزوه. 14. تأسيس ودعم برامج التعليم العالي والتدريب الذاتي.

لا نقصد هنا بإن هضة اليابان كانت بسبب ديمنج (Deming) ولكن إهتمام اليابانيون بالجودة في كل المجالات كان هو مفتاح رئيسي و وسيلة لبناء غضتها. و قد كان التعليم العالي هو الأساس في اليابان، فمنه أنطلقت التكنولوجيا وما التطور التكنولوجي الذي ولئي تشهده اليابان الا نتاج لإهتمام اليايانيين بالجودة في بحال التعليم العالي. و قد كان التهان التركيز

$$
\text { علي نشر الجودة في التعليم العالي في عدة بجالات منها: }
$$

1. تحويل الدراسة في الجامعات من إسلوب التلقين إلي التفكير والإبتكار 2. إكساب الطلاب العادات التعليمية الفعالة و تشجيع دوافعهم للتعليم العالي 3. إعداد البيئة التعليمية المنتجة و تكوينها تكوينا سليما

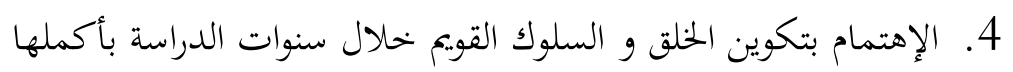
5. تكوين جهاز تعليمي محترف و ملتزم اكتسب إحترام البحتمع و أستحق المكافأة. 6. تهيئة الخدمات الوظيفية المناسبة للخريجين

و قد تمكنت اليابان من تحقيق الإنحازات نتيجة عدة عوامل منها علي سبيل الذكر

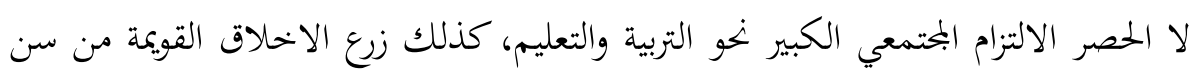
ما قبل المدرسة، و النظام المدرسي المؤثر و المدعم بمجموعة من البرامج التربوية الإضافية. 


\section{-}

الحرب الأهلية التي شهدت روندا تعد من أعنف الأزمات و أشدها قسوة، هذه الحرب بدأت مستهل أكتوبر من عام 1990 حتى عام 1994م، و هذه هنه الحرب كانت نتاج تراكمات و أحقاد قبل ذلك بعقود بين قبيلتي الهوتي والتوتسي. فبعد إستقلال روندا،

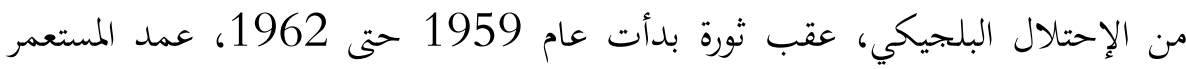
البلجيكي إلي دعم قبيلة الهوتو علي حساب بقية السكان مما خلق الأحقاد بين السكان.

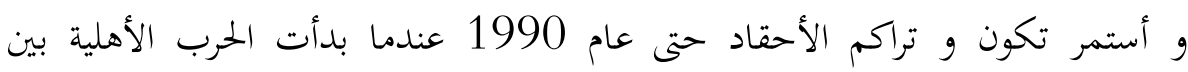
التوتسي و الهوتو. و حدثت البمازر التى سجلها التاريخ و عرفها العالم ذلك الوقت. و قد

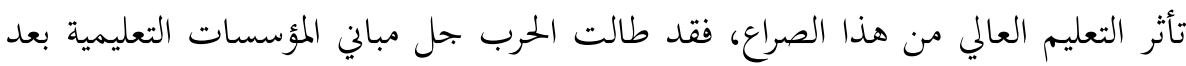

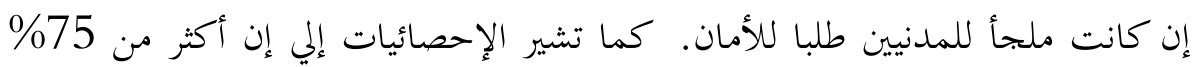
من أعضاء هيأة التدريس قتلو أو إعتقلو أو هربوا إلي خارج البلد (Hilker, 2010).

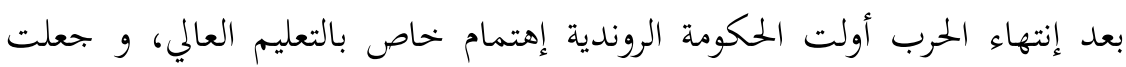

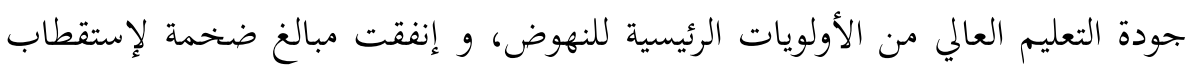
الطلاب للعودة إلي التعليم و تشجيعهم علي ذلك، (Hilker, 2010). و يشير تقرير البنك الدولي (2004) إلي إحصائيات توضح النجاح الذي أحرزته روندا في التعليم العالي

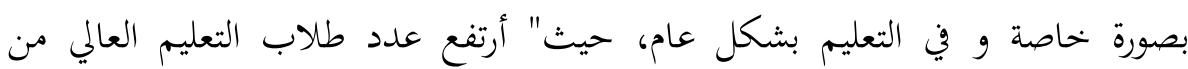

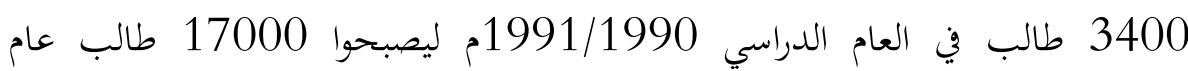
(World Bank, 2004) ، 2002/2001 و بفضل التركيز علي جودة التعليم العالي أستطعت روندا أستقطاب الشباب للإنخراط في التعليم العالي و ترك الصراع و بالتالي وصلت روندا إلي أن يشار إليها بالبنان علي إنها 
أفضل دول أفريقيا تقدما في السنوات الأخيرة. فبعد المعاناة و المذابح، عرفت روندا بإن الطريق إلي النجاح لن يكون إلا من خلال التعليم و مخرجاته و بجودة عالية.

\section{7.}

تمر ليبيا بأزمة حادة في التعليم العالي نتيجة الصراع المسلح و السياسي القائم في

البلاد. فقد تعرضت مباني المؤسسات التعليمية في ليبيا لدمار كبير في بعض المدن، كما هاجرت العديد من الكوادر العلمية إلي خارج البلاد، بالإضافة إلي نقص مستوى الإنفاق علي التعليم العالي. و قد أستفحلت أزمة التعليم العالي بإخراط شباب من مؤسيسات التعليم العالي في الصراع المسلح و ذلك من خلال مشاركتهم في الحرب القائمة. كل هذا وغيره عمق من الأزمة و زاد من تأثيراها السلبية و هزاتا الإرتدادية. فعلي سبيل المثال خسرت بعض الجامعات الليبية مكتباتما العلمية بسبب الدمار الذي طالها، و كذلك دمرت القاعات الدراسية و مدرجات المحاضرات و المعامل و المخازن العلمية وغيرها من

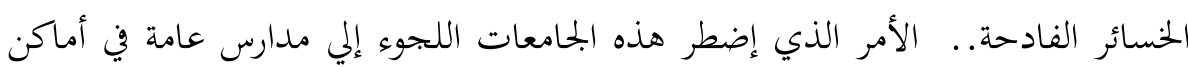

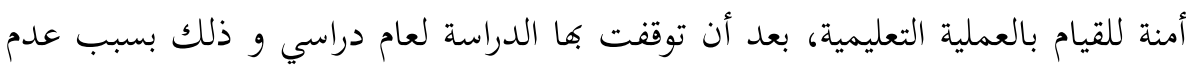
تمكنها من إستقبال الطلبة نتيجة الخوف علي حياهم و حياة العاملين بها. هذا التوقف أدى إلي تراكم مخرجات الثانويات والذين يريدون الألتحاق بالجامعة.

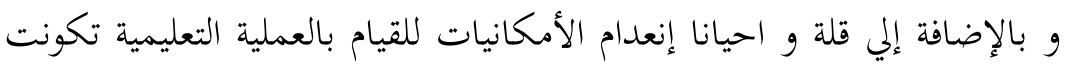
حالة نفسية سيئة لدى الطلاب، فمنهم من خسر بعض أقاربه او أصدقائه و منهم من إضطرته الحرب إلي ترك منزله و النزوح في أماكن اخرى، منعته أما من الوصول إلي جامعته او علي الأقل فقد بعض المستلزمات التي كانت عندها و يحتاجه في الدراسة كالمراجع و الملخصات وغيرها.

كما إن الحالة الإقتصادية، التي سبتها الحرب، كان لها الأثر السيء علي التعليم العالي، فتأخر مرتبات البعض وتدني مستوى العملة الليبية و إرتفاع الأسعار، نتج عنه 
صعوبة بل و أحيانا عجز الأسر على توفير الإحتياجات الأساسية لأبنائها الدارسين. و غيرها من التأثيرات الاخرى التي يعانيها التعليم العالي بسبب الأزمة القائمة.

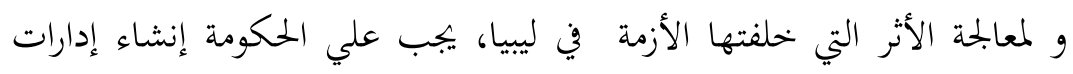
إزمة و فرق عمل فعالة لتشخيص الحالة و إقتراح الحلول الناجعة. كما يجب دعم التعليم

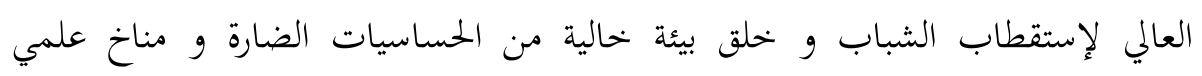
مشجع. و العمل علي إعادة بناء مادي و معنوي للتعليم العالي من خلال إعادة بناء المباني و المعامل و المكتبات، و البناء المعنوي يكون من خلال تعليم الطلاب مبادئ

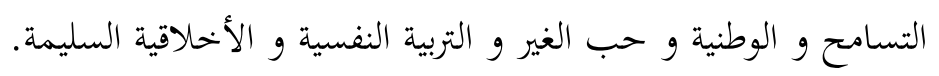

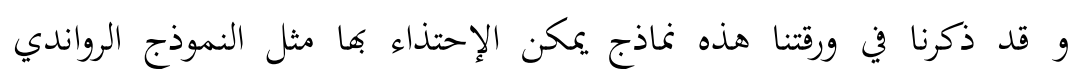
والنموذج الياباني، و ماحققتاها هاتين الدولتين من هضة علي جميع الأصعدة بسبب الإهتمام بالتعليم. كما سقنا النموذج الكولومبي و ما ألت إليه البلاد.

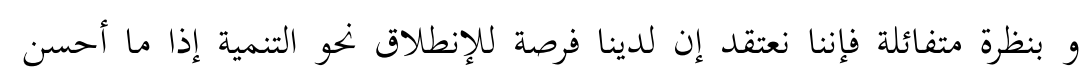
التصرف و التعامل مع قطاع التعليم العالي و العمل بجد لتأسيس تعليم عالي بجودة عالية يجذب الشباب إليه و يبعدهم عن أتون الحروب .

\section{الاستنتاجات والتوصيات}

عندما يكون التعليم العالي بجودة عالية فإنه يستقطب الشباب الذين هم وقود الحرب و يساهم في إبعادهم عن الإنتماء للجماعات المسلحة. فالتعليم العالي يلعب دور

$$
\text { مهم في إستقرار الدول التي عانت من الحروب. }
$$

قدمت هذه الورقة ثلاث نماذج الاول يوضح كيف فشلت كولومبيا في النهوض من الأزمة و ذلك بسبب إهمالها لجودة التعليم العالي، الأمر الذي قاد شباب الجامعات إلي تكوين مليشيات تحولت إلي عصابات وهاهي حتى الأن تعاني من هذا الإهمال. 
و النموذج الثاني هو اليابان، و كيف أنعكست نتائج الجودة في التعليم العالي إلي تصدر اليابان علي هرم التقدم والتطور • و النموذج الثالث هو روندا. و تأسيسا علي ذلك فإن النهوض من صدمة الأزمة يستلزم الاتي:

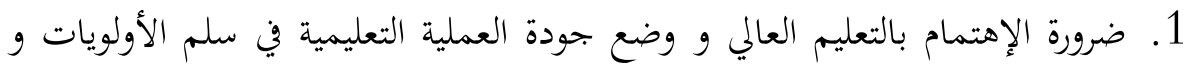
علي قمة هرم الإهتمام والرعابة.

2. التقصي عن مسببات المشاكل والمعوقات و نتائج الأزمة و إجتثاث جذورها.

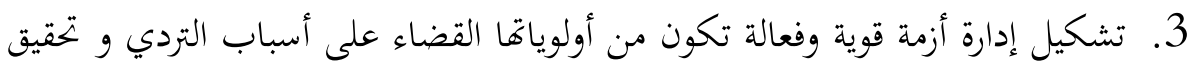

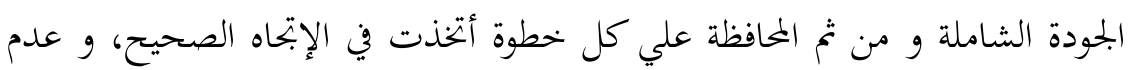
4. يجب التركيز علي أن يكون التعليم هرمي. الإصلاحات مهما كلف الأمر. 5. تشجيع الشباب علي التعلم و جعل مؤسسات التعليم العالي أماكن جذب لهم. 6. التركيز علي تحلي الطلاب بالأخلاق الحميدة و الصفات الراقية ليكونوا قدوة. 7. المحافظة علي الجودة أثناء و بعد الأزمة و العمل علي المراقبة و التقييم والتقويم المستمر.

\section{قائمة المراجع}

Crosby, P. (1979), Quality is Free, New American Library, New York.

Deming, E. (1972), Out of Crisis, Massachusetts Institute of Technology, Centre for Advanced Engineering Study, Cambridge .

Hilker, L. (2010), The Role of Education in Driving Conflict and Building Peace - The Case of Rwanda. Background paper prepared for the Education for All Global Monitoring Report 2011, UNESCO, USA 


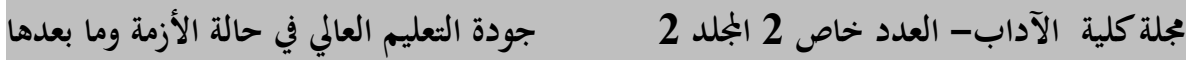

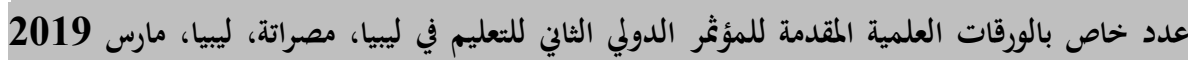

Juran, J. (1986), A Universal Approach to Managing for Quality - The Quality Trilogy", Quality Progress, August, pp.19-24.

Soler, S. (2017), Civil Conflict And Education: How Does Exposure to Civil Conflict Affect Human Capital Accumulation? Evidence From Standardized Exit Exams in Colombia. Phd. Thesis. Maastrichet University, Colombia.

Tribus, M. (1993) " TQM in education: the theory and how to put it to work", in Quality Goes to School: Readings on Quality Management in Education, American Association of School Administrators, Arlington, VA.

World Bank. (2004), The (2004) Education in Rwanda: rebalancing Resources to Accelerate Post-Conflict Devlopment and Poverty Reduction. Washington DC: The World Bank 